\title{
FUSION OF SENTINEL-1A AND LANDSAT-8 IMAGES FOR IMPROVING LAND USE/LAND COVER CLASSIFICATION IN SONGKLA PROVINCE, THAILAND
}

\author{
NUTHAMMACHOT, $\mathrm{N}^{1}{ }^{1}-$ STRATOULIAS, D. ${ }^{2,3 *}$ \\ ${ }^{I}$ Faculty of Environmental Management, Prince of Songkla University \\ P. O. Box 50 Kor-Hong, Hatyai, Songkhla 90112, Thailand \\ (e-mail: narissara.n@psu.ac.th) \\ ${ }^{2}$ Department for Management of Science and Technology Development, Ton Duc Thang \\ University, Ho Chi Minh City, Vietnam \\ ${ }^{3}$ Faculty of Applied Sciences, Ton Duc Thang University, Ho Chi Minh City, Vietnam \\ *Corresponding author \\ e-mail: dimitris.stratoulias@tdtu.edu.vn \\ (Received $3^{\text {rd }}$ Nov 2018; accepted $12^{\text {th }}$ Feb 2019)
}

\begin{abstract}
The objective of this study is to compare the performance of different data fusion techniques for improving the land use/land cover types classification accuracy in Hat Yai district, Songkla province, Thailand. SAR Sentinel-1A and optical Landsat-8 satellites are used as standalone inputs as well as to perform a data fusion based on the resolution merge and LMVM techniques. The four input datasets are classified with a supervised maximum likelihood algorithm and compared against base land cover maps; the results indicate that resolution merge of optical and SAR satellite images can significantly improve the interpretation and classification accuracy of land cover and land use types at the area of interest.
\end{abstract}

Keywords: multi-source data fusion, resolution merge, SAR, optical, LULC

\section{Introduction}

Mapping land use/land cover of large cities is a main component of detecting rapid changes from non-industrial to industrial areas. Hat Yai district hosts the fourth largest city in Thailand undergoing rapid and fundamental landscape changes leading to increased urbanization. Therefore, mapping land use/land cover holds an important role in monitoring and assessing environmental changes, land use planning and decisionmaking activities (Dengsheng et al., 2012). Remote sensing is an effective technology for analysing land use/land cover change in attributes such as crop, forest and agriculture. South Thailand is located in the tropical climatic zone and is frequented by cloud coverage which is an impediment for passive microwave and optical sensors; active microwave sensors, such as Synthetic Aperture Radar (SAR), on the other hand, are weather independent systems. A combination of radar and optical data integrates complimentary information from two fundamentally different systems and improves land use/land cover classification accuracy (Sukawattanavijit, 2015). Image fusion is an active field of research in remote sensing (for a practical guide the reader is directed to Pohl and Genderen, 2016) and other application areas such as medical imaging and night vision (Li et al., 2017).

Several studies have attempted to fuse satellite data. For instance, Sukawattanavijit (2015) classified land cover in Wang Muang district located at the northern part of Saraburi Province, central Thailand by using RADARSAT-2 and Landsat- 8 satellite 
images with wavelet-based fusion. The results showed that the fused image had higher accuracy compared to the single radar data. Dimov et al. (2016) found that the Ehlers fusion is the best technique when looking at crop areas in Fergana Valley in Uzbekistan. In another study Chen et al. (2017) investigate the performance of multi-source remotely sensed data fusion in the context of improving land cover classification; they found that the fused data, which integrated temporal, spectral, angular, and topographic features, achieved a higher mapping accuracy for land cover classification in comparison to the original data sources. Salman et al. (2017) demonstrated that fused dataset provided accuracy $99.6 \%$ of land use/land cover classification using machine vision. Similarly, Sukawattanavijit et al. (2017) suggested that genetic algorithms and Support Vector Machines (SVMs) lead to improve classification of up to $95 \%$ of different land cover types when fusing SAR RADARSAT-2 data and optical Thaichote (THEOS) data. In another case study, Puttinaovarat and Horkaew (2017) investigated the extraction of buildings from fused satellite images and on the basis of spectral indices instead of reflectance bands. It is worth noting, however, that there are other studies which mention that combining optical and SAR data does not guarantee improvement compared with using single data source for urban land cover classification (e.g. Zhang and $\mathrm{Xu}, 2018$ ).

The objective of this study is to evaluate the capability of SAR Sentinal-1, optical Landsat- 8 and their fusion resulting from two independent techniques for extracting land use/land cover information via supervised classification in Hat Yai district, Songkla province, Thailand.

\section{Experimental}

\section{Study area}

Hat Yai district is located in South Thailand, situated at $7^{\circ} 1^{\prime} \mathrm{N}$ latitude $100^{\circ} 28^{\prime} \mathrm{E}$ longitude, and encompasses the country's fourth largest city. It covers a total area of approximately $852.796 \mathrm{~km}^{2}$ and hosts a population of over 800,000 inhabitants. The climatic conditions of this tropical area are hot and humid. The dry season lasts from January to April and the wet season from May to December. Hat Yai is a commercial district with the predominant activities being tourism, fishing industries, agriculture, rubber and oil palm plantations (Appianing et al., 2016). The study area is depicted in Figure 1.

\section{SAR data}

Sentinel-1A is a SAR satellite providing medium and high resolution imagery at Cband (centre frequency: $5.405 \mathrm{GHz}$ ) under all-weather conditions. The C-SAR is useful for land and marine monitoring (ESA, 2016). In this study, a Sentinel-1A image with $\mathrm{VV} / \mathrm{VH}$ dual polarization was used. The spatial resolution is $5 \times 20 \mathrm{~m}$ and was acquired at Level-1 Ground Range Detected (GRD) mode with $250 \mathrm{~km}$ swath, multilook intensity only and Interferometric Wide (IW) swath mode. The specifications of Sentinel-1A are presented in Table 1.

\section{Optical data}

The Landsat-8 Operational Land Imager (OLI) image was acquired on 18th March 2015. Landsat- 8 has 11 spectral bands with a spatial resolution of 15,30 and $100 \mathrm{~m}$ for 
the panchromatic, multispectral and thermal bands respectively. The specific image was selected based on the relatively low cloud coverage $(<10 \%)$. Landsat -8 band specifications are presented in Table 1.

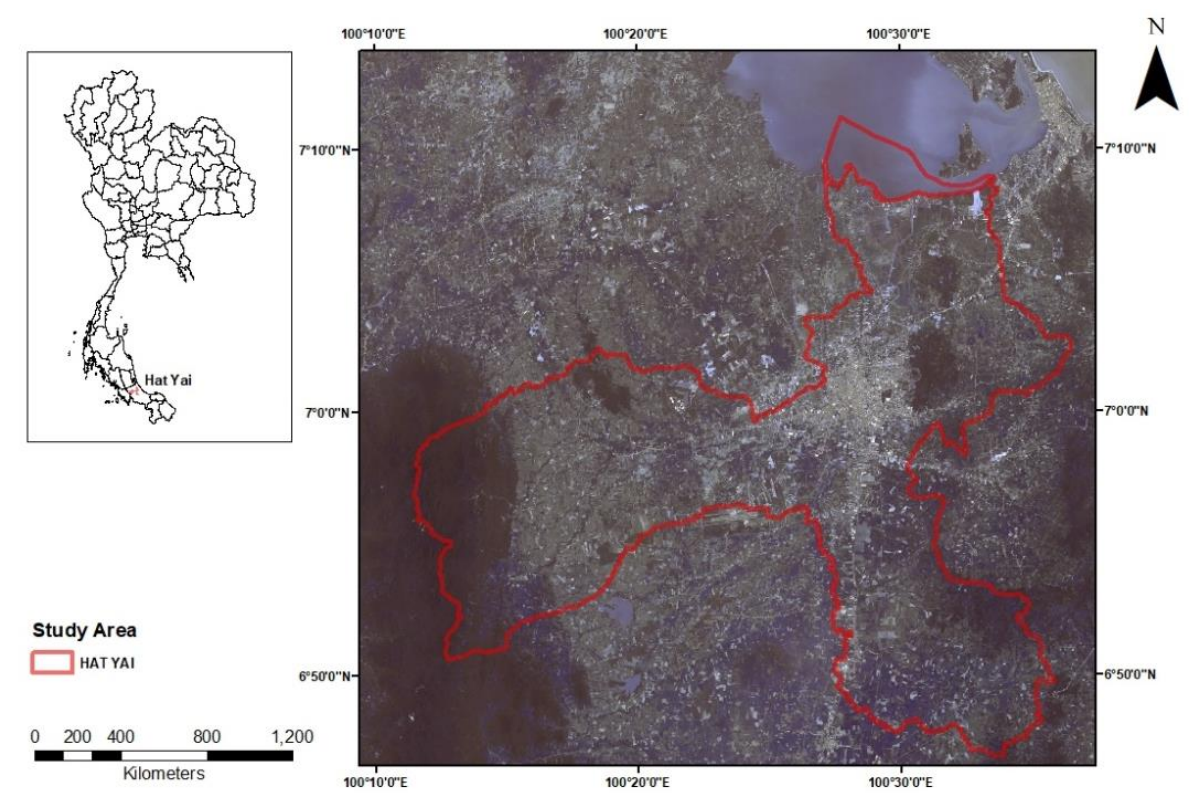

Figure 1. Map of the location of the study area: Hat Yai district, Songkla province, Thailand

Table 1. Sentinel-1A and Landsat-8 OLI image specifications

\begin{tabular}{c|c|c}
\hline Specifications & Sentinel-1A & Landsat-8 OLI \\
\hline Acquisition date & $25^{\text {th }}$ February 2015 & $18^{\text {th }}$ March 2015 \\
\hline $\begin{array}{c}\text { Acquisition time } \\
\text { (hh:mm) }\end{array}$ & $23: 02$ (UTC) & $03: 33$ (UTC) \\
\hline Imaging mode & IW swath mode & OLI and TIRS \\
\hline Polarization & VV/VH dual polarization & - \\
\hline Data product & Level-1 ground range detected mode & Level 1 Terrain \\
\hline Resolution mode & $20 \mathrm{~m}$ (full resolution) & 30 m (except panchromatic (15 m) and \\
& & $0.43-0.45$ (Coastal Aerosol) \\
& & $0.45-0.51$ (Blue) \\
& & $0.53-0.59$ (Green) \\
& & $0.64-0.67$ (Red) \\
& & $0.85-0.88$ (Near Infrared NIR) \\
Bands & C-band (5.4 GHz) & $1.57-1.65$ (SWIR 1) \\
& & $2.11-2.29$ (SWIR 2) \\
& & $0.50-0.68$ (Panchromatic) \\
& & $1.36-1.38$ (Cirrus) \\
& & $10.60-11.19$ (Thermal Infrared - TIRS 1) \\
& & \\
\hline
\end{tabular}

\section{Ground truth data}

Auxiliary data were provided by the GEO-Informatics Research Centre for Natural Resources and Environment and the Southern Regional Centre of Geo-Informatics and 
Space Technology, Thailand. Figure 2 depicts the map produced in 2015 (the same year with the two satellite image acquisitions) encompassing land use/land cover classes of urban area, paddy fields, plantations (fruits, palm oil and rubber), forests and lake. Land cover has been classified using high resolution satellite images and ground data.

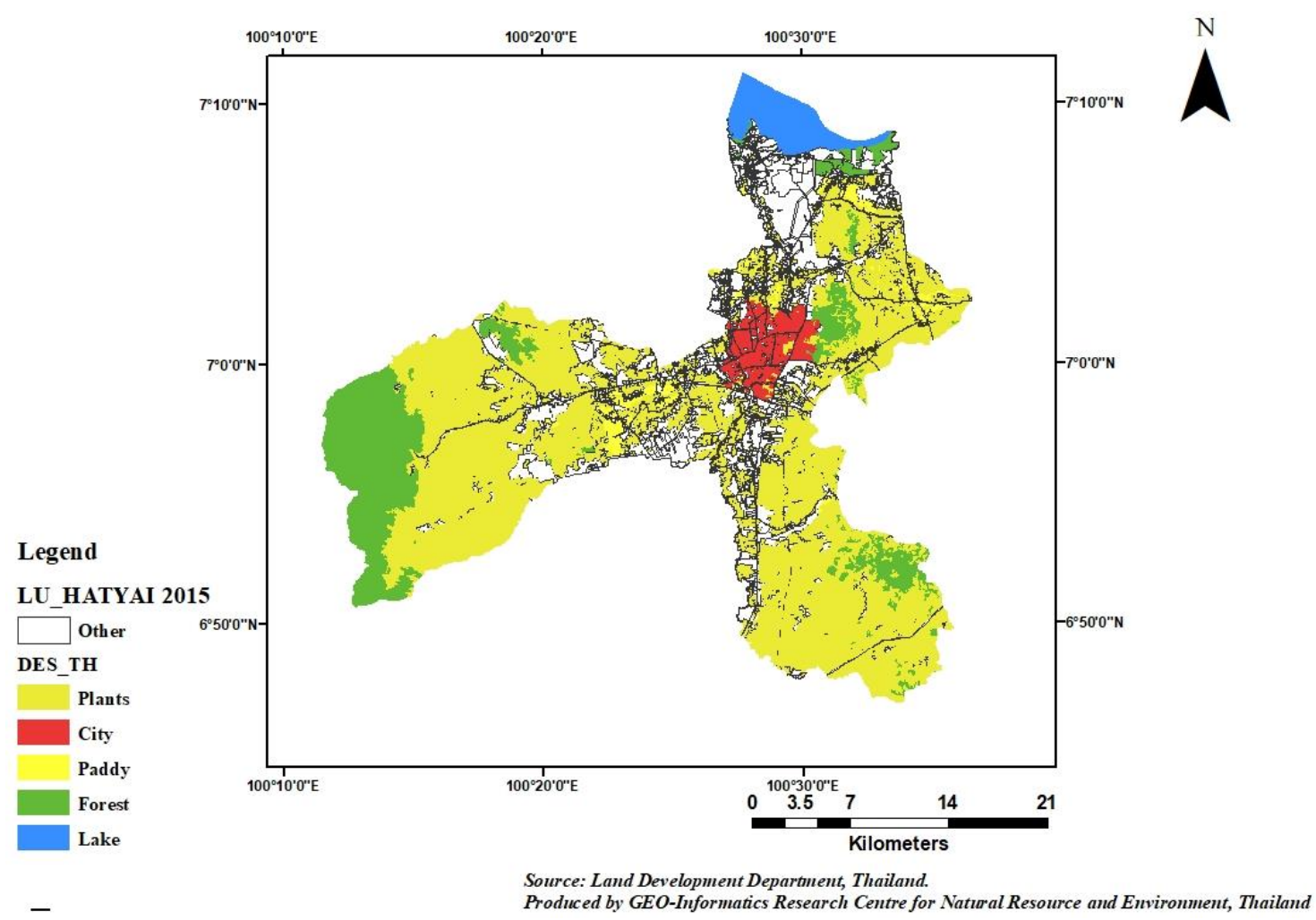

Figure 2. The auxiliary data from the Land Development Department, Thailand. (The data were taken with the permission of the Land Development Department, Thailand. Produced by the GEO-Informatics Research Centre for Natural Resources and Environment Southern Regional Centre of Geo-Informatics and Space Technology, Thailand 2015)

\section{Methodology}

\section{Pre-processing}

The Sentinel-1A image at Level-1 GRD mode was first calibrated so that the pixel values represent the radar backscatter of the reflecting surface. Subsequently, the multilook of the SAR image was generated by averaging over the range and azimuth resolution cells to improve the radiometric resolution. A GammaMAP $5 \times 5$ window size was applied to reduce speckle noise. The Shuttle Radar Topography Mission (SRTM) 3sec Digital Elevation Model (DEM) was used for the terrain correction. Finally, the Sentinel-1A backscattered values were converted to decibel (dB) as shown in Figure $3 a$.

The Landsat-8 OLI image was converted from Digital Numbers (DN) to Top-OfAtmosphere (TOA) reflectance with the ENVI 5.1 software. The Fast Line-of-sight Atmospheric Analysis of Spectral Hypercube (FLAASH) atmospheric correction method was used for retrieving the Top-Of-Canopy (TOC) spectral reflectance from the Landsat-8 OLI image as depicted in Figure $3 b$. 


$$
-3127-
$$

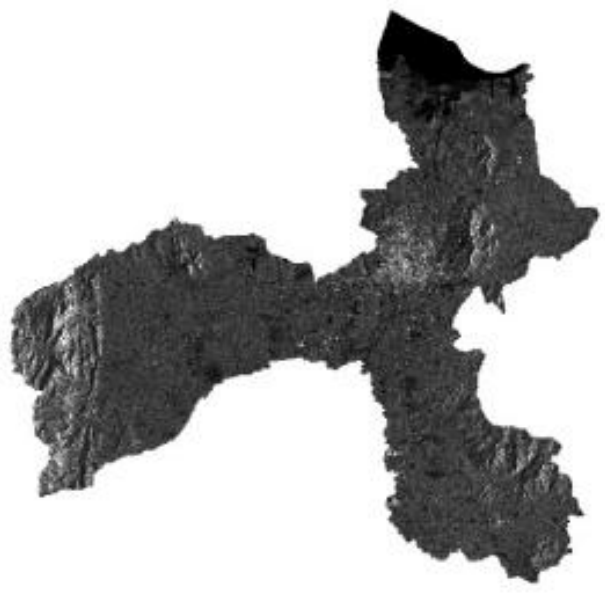

a.

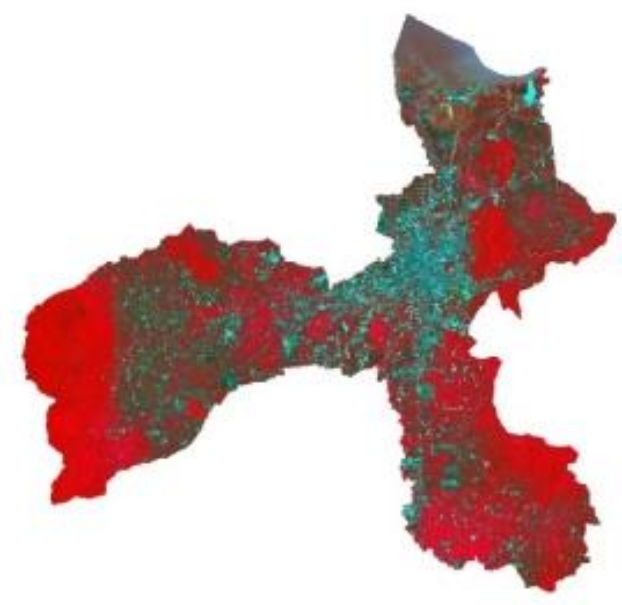

C.

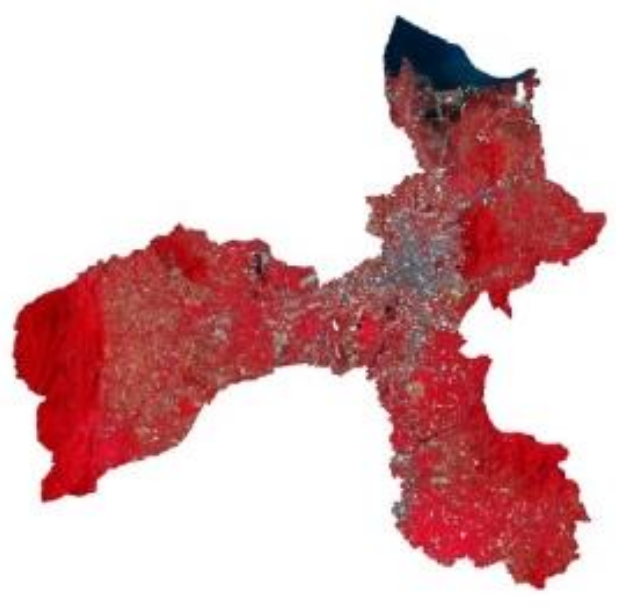

b.

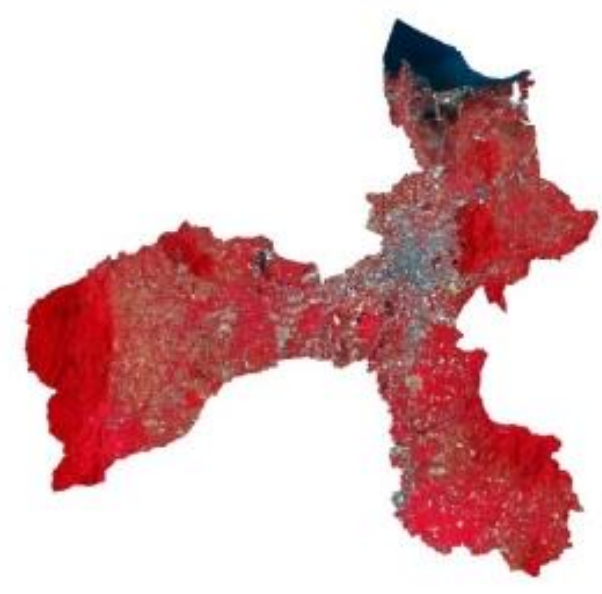

d.

Figure 3. The four input layers in the classification. a) Sentinel-1A, b) Landsat-8 (5-4-3), c) resolution merge, d) $L M V M$

\section{Data fusion}

The VV/VH polarized $\mathrm{C}$ band Sentinel-1A image and a layer stack of three spectral bands (i.e. 5, 4, 3) from the Landsat-8 image were used for the two fusion methodologies. The Local Mean Variance Matching (LMVM) and the Resolution merge data fusion techniques were applied in this study and the final result is presented in Figure $3 c$ and $d$.

The LMVM is a robust algorithm for fusing multispectral and panchromatic images of the same sensor (Nikolakopoulos, 2008). In this study, it was used to integrate the two images $(E q .1)$, namely a high spatial resolution SAR Sentinel-1 A image with spectral information from a low spatial resolution multispectral Landsat-8 image (Chinsu et al., 2015; Zhang, 2004; Wald et al., 1997; Ranchin and Wald, 2000). 


$$
F_{K(i, j)}=P_{(i, j)} \times \frac{M_{k(i, j)(w, h)}}{P_{(i, j)(w, h)}}
$$

where:

$F_{K(i, j)}=$ the fused image

$P_{(i, j,}=$ the high spatial resolution images at pixel coordinates $(\mathrm{i}, \mathrm{j})$

$M_{k(i, j,}=$ the low spatial resolution images at pixel coordinates $(\mathrm{i}, \mathrm{j})$

$M_{k(i, j)(w, h)}=$ the local means calculated inside the window of size $(\mathrm{w}, \mathrm{h})$

$P_{(i, j)(w, h)}=$ the local means calculated inside the window of size $(\mathrm{w}, \mathrm{h})$

The resolution merge combines images of differing spatial resolutions. VV/VH polarized $\mathrm{C}$ band Sentinel-1A image and three bands (i.e. 5, 4, 3) of the Landsat-8 multispectral image were merged together into a common image file format. This step prepares the fused multi-sensor data for classifications as in Figure $3 c$ (Erdogan et al., 2008; Vaiopoulos et al., 2001).

\section{Image classification}

We classified the input layers using the maximum likelihood algorithm in the 5 main land cover / land use classes of the study area; namely forest, paddy rice, plantations, urban, lake, and unclassified. Ground truth and test data were collected from the Land Development Department, Thailand. A cumulatively large training and validation set for each representative class was selected based on the ground truth dataset to train and validate the classifier (Table 2).

Table 2. Training and validation sets size used in the 4 classification scenarios

\begin{tabular}{c|c|c}
\hline Class & Training set (number of pixels) & Validation set (number of pixels) \\
\hline Lake & 1345 & 920 \\
Forest & 569 & 539 \\
City & 264 & 934 \\
Paddy & 1247 & 464 \\
Plants & 232 & 575 \\
\hline
\end{tabular}

\section{Accuracy assessment}

The accuracy of the image classification was assessed against the reference data with a confusion matrix (Congalton, 1991). The main measures of comparison were the overall accuracy and the kappa coefficient of agreement $(\kappa)$. Overall accuracy is the total number of correctly classified samples (i.e. the diagonal cells in the confusion matrix) divided by the total number of reference pixels. In essence, it presents the probability that a sample will be correctly classified by the algorithm. $\kappa$ is a measure of the correctly classified samples adjusted to the amount of agreement that could be expected only due to chance. $\kappa$ utilizes all elements from the confusion matrix by taking into account both errors of commission and omission, and thus provides a more complete picture of the information comprising the confusion matrix than the overall accuracy (Napoleon et al., 2013). The Kappa statistics and the overall accuracy assessment were calculated to compare the accuracy of the classified images. 


\section{Statistical analysis}

Two statistical moments, namely the mean and the standard deviation (square root of the variance), are used as evaluation indicators to judge on the ability to use the two fusion image techniques. A low standard deviation is regarded as an indicator of higher class separability (Napoleon et al., 2013).

The mean is obtained by dividing the sum of observed values by the number of observations, $\mathrm{N}$ as in Equation 2:

$$
\bar{X}=\frac{\sum f X}{N}
$$

where $f$ is the probability of the occurrence of the value of $X$.

The standard deviation is a measurement of the variation or dispersion from the average. A low standard deviation indicates that the data points tend to be very close to the mean (the so called expected value); a high standard deviation indicates that the data points are spread out over a large range of values. The standard deviation is calculated according to Equation 3:

$$
S t d=\sqrt{\frac{\sum(X-\bar{X})^{2}}{N}}
$$

where:

Std is standard deviation,

$X$ is sample,

$\bar{X}$ is sample mean,

$\mathrm{N}$ is number of score in sample.

\section{Validation}

Validation was engaged to assess the performance of this model. Lu et al. (2016) stated that $\mathrm{R}^{2}$ is commonly used to validate a model. Two groups of data were chosen, namely observed and predicted. Observed data were derived from land-use ground truth data (Land Development Department, Thailand) and predicted data were obtained from the resolution merge image classification. We selected 5 land use types (city, forest, lake, paddy, plants and others) from observed data and resolution merge image classification for the validation. The linear regression was estimated to validate the model and the reliability was judged upon the high $\mathrm{R}^{2}$.

\section{Results and discussion}

The results for the standalone classification of the Sentinel-1A SAR image indicate an overall accuracy of $58.50 \%$ and a kappa coefficient of 0.48 while for the Landsat- 8 optical image an overall accuracy of $67.16 \%$ and a kappa coefficient of 0.59 is observed (Table 3). The individual SAR image is able to clearly extract the water class (i.e. lake). However, the extraction of other classes is not so accurate; especially at the hilly parts of the study area misclassifications occur for the vegetation classes as the forested, 
plants and largely the urban areas are underestimated. Moreover, there are high overlaps with paddy and plants classes. The reason might be attributed to the fact that the speckle noise and the mixed pixel problem cannot be entirely eliminated. The Landsat- 8 optical image provides a better representation of the forest class in comparison to the Sentinel-1 SAR image. However the urban class is overestimated in comparison to the base map. In the case of the classification of the fused products, the resolution merge provided an overall accuracy of $79.75 \%$ and a kappa coefficient of 0.75 which is higher than the individual satellite images while LMVM provided an overall accuracy of 59.84\% and a kappa coefficient of 0.52. LMVM classification includes many unclassified pixels and its overall accuracy of $59.84 \%$ and kappa coefficient of 0.52 are similar to the performance of the standalone Sentinel-1A classification (Table 3). LMVM has been suggested as a robust algorithm when fusing multispectral and panchromatic images (Nikolakopoulos, 2008), however, in our case of merging multi-source data it does not offer any significant advantage. The thematic maps of the classification results are presented in Figure 4.

Resolution merge resulted to the most accurate representation with producer accuracy of the lake area being 83.91 while the user accuracy is $99.61 \%$. The producer accuracies of forest, city, plants and paddy areas are $72.17 \%, 86.94 \%, 62.09 \%$ and $87.72 \%$, respectively while the user accuracies for the above-mentioned classes are $99.49 \%, 88.65 \%, 64.67 \%$ and $57.73 \%$, respectively. Apart from the highest classification accuracy, resolution merge is the result with the best visual agreement with the base map (Fig. 2). Our findings are in agreement with other studies which have concluded that the classification accuracy is increased after fusing data from separate satellite sources in comparison to stand-alone classification of a images from a single satellite sensor (e.g. Chen et al., 2016).

From overall classification of the Landsat-8, resolution merge and LMVM classified images we found that lake and forest recorded almost $100 \%$ classification accuracy while urban, plants and paddy showed the lower percentages accuracies of land use and land cover classes. This might be explained by the fact that the forest in dominated by dense evergreen and high-density trees. Plants and paddy were confused between each other and, consequently, this decreased the reliability of their accuracies when compared to other land use and land cover types classified (Nwaogu et al., 2017; Johnson et al., 2016). Another reason, might be that some areas from the secondary data were attributed another class in comparison to the classification of the satellite images in land use types (lake, forest, city, plants and paddy).

Table 3. The overall classification accuracy of the classified images. PA and UA refer to producer's and user's accuracy, respectively. All values are in percentages (\%)

\begin{tabular}{c|c|c|c|c|c|c|c|c}
\hline \multirow{2}{*}{ Land cover types } & \multicolumn{2}{|c|}{ Sentinel-1A } & \multicolumn{2}{c|}{ Landsat-8 } & \multicolumn{2}{c|}{ Resolution merge } & \multicolumn{2}{c}{ LMVM } \\
\cline { 2 - 9 } & PA & UA & PA & UA & PA & UA & PA & UA \\
\hline Lake & 100.00 & 100.00 & 65.76 & 100.00 & 83.91 & 99.61 & 44.89 & 100.00 \\
Forest & 31.17 & 36.21 & 61.22 & 100.00 & 72.17 & 99.49 & 52.50 & 100.00 \\
City & 27.73 & 59.13 & 91.86 & 77.58 & 86.94 & 88.65 & 89.94 & 88.05 \\
Plants & 67.65 & 37.33 & 51.13 & 75.58 & 62.09 & 64.67 & 41.57 & 80.74 \\
Paddy & 58.62 & 49.19 & 46.98 & 39.21 & 87.72 & 57.73 & 60.13 & 42.92 \\
\hline Overall accuracy & \multicolumn{2}{|c|}{58.50} & \multicolumn{2}{|c|}{67.16} & \multicolumn{2}{c|}{79.75} & 59.84 \\
\hline Kappa coefficient & \multicolumn{2}{|c|}{0.48} & \multicolumn{2}{|c|}{0.59} & \multicolumn{2}{c|}{0.75} & 0.52 \\
\hline
\end{tabular}




$$
\text { - } 3131-
$$

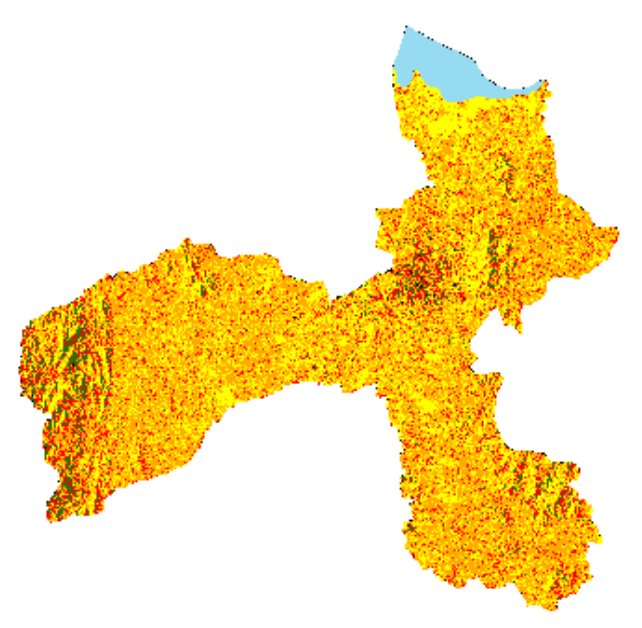

A
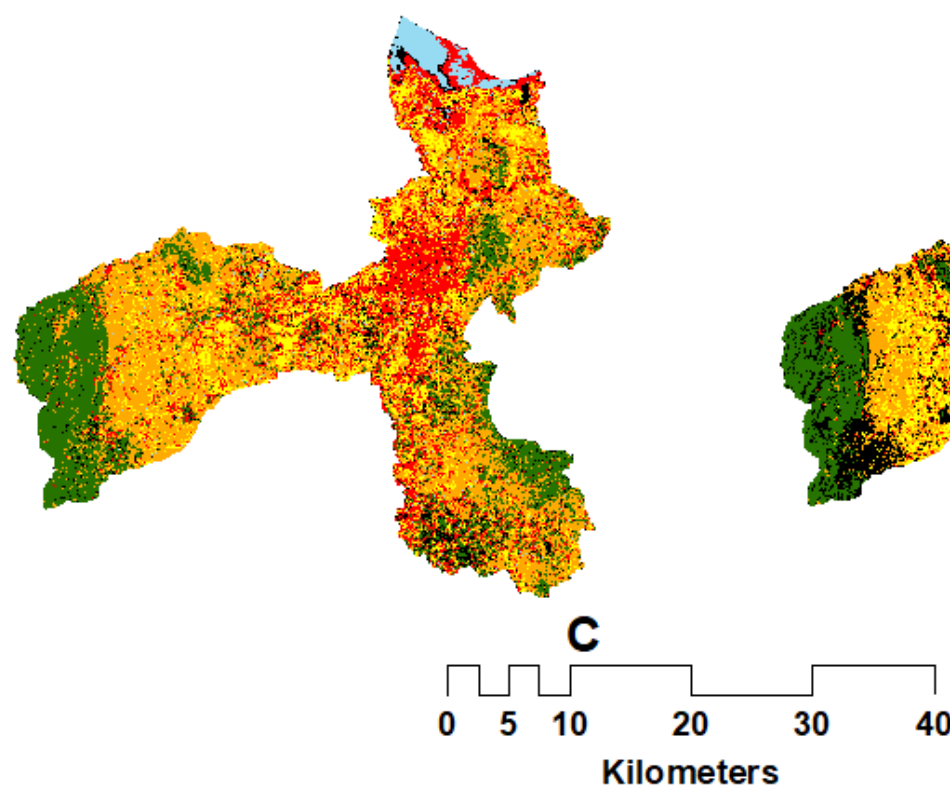

Figure 4. Thematic maps produced from the maximum likelihood classification of the four input datasets: Sentinel-1A (A), Landsat-8 (B), Resolution merge (C) and LMVM (D)

From the statistical analysis of the mean and the standard deviation as presented in Table 4, it is obvious that the highest standard deviation is 2.13 for Sentinel-1 and followed by 1.76 for Landsat-8, 1.72 for LMVM and 0.94 for resolution merge. As a good performance (i.e. higher class separability as suggested by Napoleon et al., 2013) is indicated by a low standard deviation, it is clear that resolution merge fusion technique perform the best in this study. 


$$
\text { - } 3132 \text { - }
$$

Table 4. The mean and standard deviation of the 4 input layers

\begin{tabular}{c|c|c}
\hline Image & Mean & Standard deviation \\
\hline Sentinel-1A & 1.62 & 2.13 \\
Landsat-8 & 1.39 & 1.76 \\
Resolution merge & 2.75 & 0.94 \\
LMVM & 0.98 & 1.72 \\
\hline
\end{tabular}

The validation exercise resulted to Table 5 and Figure 5 and suggests that the correlation between the observed and predicted model gave a moderate $\mathrm{R}^{2}$. This means that approximately $52 \%$ of the observed values could be explained by the predicted model. The linear regression analysis and the equation describing it are presented in Figure 5, where $\mathrm{y}$ is predicted value and $\mathrm{x}$ is observed value. The reason $\mathrm{R}^{2}$ value resulted to moderate level is because the land use map from the predicted model were indicated otherwise as in Table 5. For example, the land use type of "others" was classified around $23.22 \%$ while predicted resolution merge resulted to just $48 \%$ in this case.

Table 5. Statistics of the observed and predicted model for the resolution merge input

\begin{tabular}{c|c|c|c|c}
\hline $\mathbf{L U}$ & $\begin{array}{c}\text { Observed } \\
\left(\mathbf{a r e a} \mathbf{~ k m}^{\mathbf{2}}\right)\end{array}$ & $\begin{array}{c}\text { Observed } \\
\mathbf{( \% )}\end{array}$ & $\begin{array}{c}\text { Predicted resolution merge } \\
\left(\mathbf{a r e a ~}_{\mathbf{~ k m}}^{\mathbf{2}}\right)\end{array}$ & $\begin{array}{c}\text { Predicted } \\
(\mathbf{\%})\end{array}$ \\
\hline City & 26 & 3.16 & 154 & 19.09 \\
Forest & 112 & 13.89 & 161 & 19.94 \\
Lake & 28 & 3.40 & 22 & 2.77 \\
Paddy & 15 & 1.86 & 115 & 14.19 \\
Plants & 441 & 54.47 & 308 & 38.04 \\
Others & 188 & 23.22 & 48 & 5.98 \\
Total & 809 & 100.00 & 809 & 100.00 \\
\hline
\end{tabular}

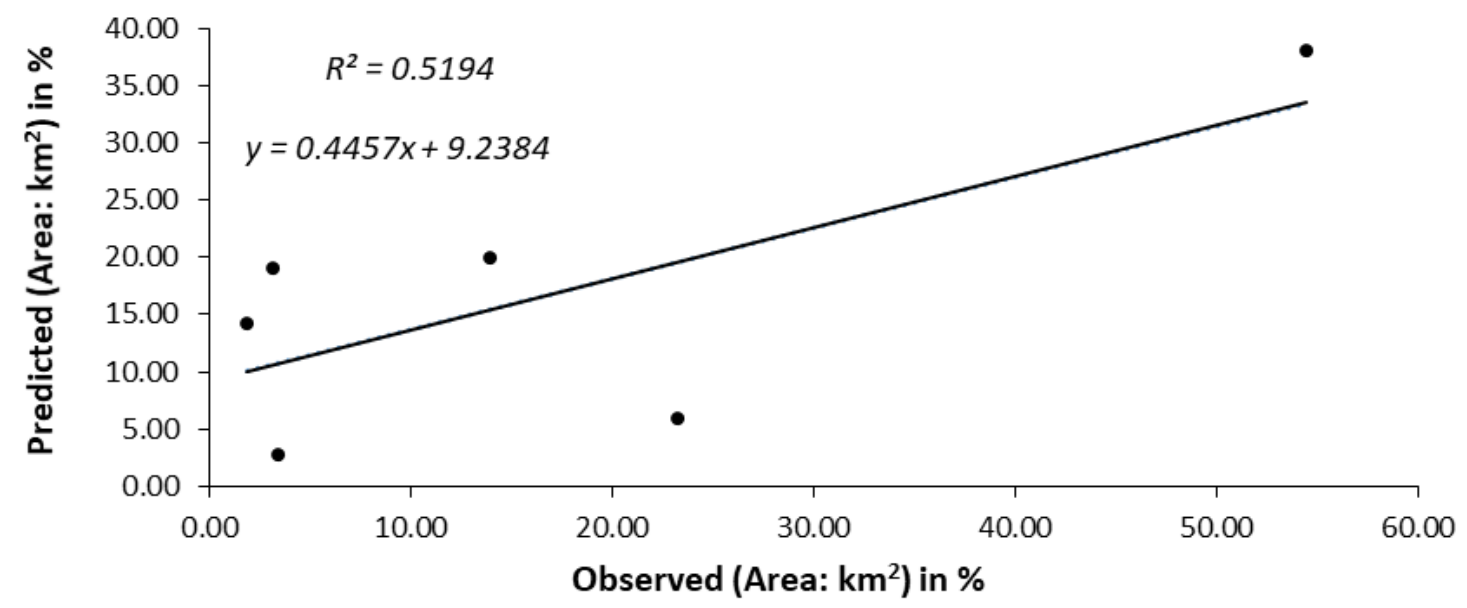

Figure 5. Scatter plot of observed values and predicted model 


\section{Conclusions}

The present study classified land use in Hat Yai in the year 2015 using two schemes; first the dual polarization Sentinel-1A and the Landsat-8 images standalone, therefore based solely on the SAR or optical information respectively; second, the Sentinel-1A was fused with the Landsat- 8 image in an attempt to combine complimentary information acquired from the SAR and optical sensors. Resolution merge and LMVM techniques were used to fuse images in the latter case. The maximum likelihood supervised classification algorithm was used for the classification of the four experiments. The outcome $\mathrm{VV} / \mathrm{VH}$ dual polarization image did not result to confident results. However, when combining an optical Landsat-8 image with a dual polarized (VV/VH) Sentinel-1A image, the results indicated that the images conserved the spectral characteristics in the original Landsat- 8 data and also improved the spatial information. The fusion of a Sentinel-1A dual polarization image with a Landsat-8 image with the resolution merge technique outperformed the LMVM technique according to calculated statistical results. Our study strongly suggests that the most accurate and realistic classification scheme tested is the resolution merge fusion of the optical and SAR data. We showcased that the synergistic use of SAR and optical satellite data greatly improve the land cover and land use classification accuracy, in accordance with other studies in the literature (e.g. Otukei et al., 2015). In the future, it is recommended to consider other land use and land cover areas in the analysis and also use other SAR satellite images to investigate the impacts of different SAR input in the fusion outputs. In addition to that, image classification processes could be applied to evaluate the impacts of fusion algorithms over land use map production.

Acknowledgements. The authors would like to thank GEO-Informatics Research Center for Natural Resource and Environment and Southern Regional Center of Geo-Informatics and Space Technology for their help and support by making available the auxiliary data. We would also like to thank the Faculty of Environmental Management, Prince of Songkla University for providing funding in support of this research.

\section{REFERENCES}

[1] Appianing, C. B., Kongchouy, N., Thinnukool, O. (2016): Land use change and development modeling using logistic regression. - Journal of Engineering and Applied Sciences 11: 1676-1681.

[2] Chen, B., Huang, B., Xu, B. (2017): Multi-source remotely sensed data fusion for improving land cover classification. - ISPRS Journal of Photogrammetry and Remote Sensing 124: 27-39.

[3] Chinsu, L., Chao-Cheng, W., Khongor, T., Yen-Chieh, O., Chein-I. C. (2015): Effects of atmospheric correction and pansharpening on LULC classification accuracy using WorldView-2 imagery. - Information Processing in Agriculture 2015(2): 25-36.

[4] Congalton, R. G. (1991): A review of assessing the accuracy of classifications of remotely sensed data. - Remote Sensing of Environment 37(1): 35-46.

[5] Dengsheng, L., Qi, C., Guangxing, W., Emilio, M., Mateus, B., Maozhen, Z., Gaia, V. L., David, S. (2012): Aboveground forest biomass estimation with Landsat and LiDAR data and uncertainty analysis of the estimates. - International Journal of Forestry Research 6: $1-16$.

[6] Dimov, D., Kuhn, J., Conrad, C. (2016): Assessment of cropping system diversity in the Fergana Valley through image fusion of Landsat 8 and SENTINEL-1. - ISPRS Annals of 
Photogrammetry, Remote Sensing and Spatial Information Sciences III-7: 173-180. DOI: 10.5194/isprsannals-III-7-173-2016.

[7] Erdogan, M., Maras, H. H., Yilmaz, A., Özerbil, Ö. T. (2008): Resolution merge of 1:35.000 scale aerial photographs with LANDSAT 7 ETM imagery. - The International Archives of the Photogrammetry, Remote Sensing and Spatial Information Sciences XXXVII(Part B7): 1281-1286.

[8] ESA (2016): Sentinel-1 Satellite. - https://earth.esa.int/web/guest/missions/esaoperational-eo-missions/sentinel-1 (accessed on 23 April 2018).

[9] Johnson, B. A., Iizuka, K. (2016): Integrating OpenStreetMap crowdsourced data and Landsat time-series imagery for rapid land use/land cover (LULC) mapping: Case study of the Laguna de Bay area of the Philippines. - Applied Geography 67: 140-149.

[10] Li, S., Kang, X., Fang, L., Hu, J., Yin, H. (2017): Pixel-level image fusion: a survey of the state of the art. - Information Fusion 33: 100-112.

[11] Lu, D., Chen, Q., Wang, G., Liu, L., Li, G., Moram, E. (2016): A survey of remote sensing-based aboveground biomass estimation methods in forest ecosystems. - Journal International Journal of Digital Earth 9: 63-105.

[12] Napoleon, D., Mageshwari, V., Revathi, P. (2013): A resourceful filtering technique for texture segmentation and enhancement in remote sensing images using morphological operations. - International Journal of Research in Advent Technology 1: 223-228.

[13] Nikolakopoulos, K. G. (2008): Comparison of nine fusion techniques for very high resolution data. - Photogrammetric Engineering \& Remote Sensing 74(5): 647-659.

[14] Nwaogu, C., Okeke, O. J., Fadipe, O. O., Bashiru, K. A., Pechanec, V. (2017): Is Nigeria losing its natural vegetation and landscape? Assessing the landuse-landcover change trajectories and effects in Onitsha using remote sensing and GIS. - Open Geoscience 9: 707-718.

[15] Otukei, J. R., Blaschke, T., Collins, M. (2015): Fusion of TerraSAR-x and Landsat ETM+ data for protected area mapping in Uganda. - International Journal of Applied Earth Observation and Geoinformation 38: 99-104.

[16] Pohl, C., Van, G. J. (2016): Remote Sensing Image Fusion: A Practical Guide. - CRC Press, Baton Rouge, FL.

[17] Puttinaovarat, S., Horkaew, P. (2017): Urban areas extraction from multi sensor data based on machine learning and data fusion. - Pattern Recognition and Image Analysis 27(2): 326-337.

[18] Ranchin, T., Wald, L. (2000): Fusion of high spatial and spectral resolution images: the ARSIS concept and its implementation. - Photogramm Eng Remote Sens 66(1): 49-61.

[19] Salman, Q., Dost, M. K., Syed, F. Q., Abdul, R., Nazir, A., Mutiullah, J., Ali, N. S., Syed, S. M., Khalid, S., Sarfraz, A. A. (2017): Multisource data fusion framework for land use/landcover classification using machine vision. - Journal of Sensors 4: 1-8.

[20] Sukawattanavijit, C. (2015): Fusion of RADARSAT-2 imagery with LANDSAT-8 multispectral data for improving land cover classification performance using SVM. 2015 IEEE 5th Asia-Pacific Conference on Synthetic Aperture Radar (APSAR): 284-288.

[21] Sukawattanavijit, C., Chen, J., Zhang, H. (2017): GA-SVM algorithm for improving land-cover classification using SAR and optical remote sensing data. - IEEE Geoscience and Remote Sensing Letters 14(3): 284-288.

[22] Vaiopoulos, D. A., Nikolakopoulos, K., Skianis, G. A. (2001): A comparative study of resolution merge techniques and their efficiency in processing images of urban areas. Remote Sensing and Data Fusion over Urban Areas, IEEE/ISPRS Joint Workshop 2001, pp. 270-274.

[23] Wald, L., Ranchin, T., Mangolini, M. (1997): Fusion of satellite images of different spatial resolutions: assessing the quality of resulting images. - Photogramm Eng Remote Sens. 63(6): 691-9. 


$$
\text { - } 3135 \text { - }
$$

[24] Zhang, H., Xu, R. (2018): Exploring the optimal integration levels between SAR and optical data for better urban land cover mapping in the Pearl River Delta. - International Journal of Applied Earth Observation and Geoinformation 64: 87-95.

[25] Zhang, Y. (2004): Understanding image fusion. - Photogramm Eng Remote Sens 70(6): 657-61. 